\title{
Advances in High-Resolution EBSD: Extracting Further Details from Kikuchi Patterns
}

\author{
J.A. Basinger,* D.T. Fullwood,* B.L. Adams, ${ }^{*}$ C. Sorensen,* S.R. Niezgoda** \\ * Department of Mechanical Engineering, Brigham Young University, 435 CTB, Provo, UT 84602 \\ ** Materials Science and Technology Division, Los Alamos National Laboratory, Los Alamos, New \\ Mexico 87545
}

Orientation imaging methods have been a mainstay of characterization efforts for crystalline materials for nearly two decades. Electron backscatter diffraction (EBSD) images provide statistical data regarding atomic alignment to create relatively large scale (up to several millimeters) orientation maps in crystalline and polycrystalline materials. However, recent work has highlighted that the information in the diffraction images has generally been under-used. Advances in accessing this often-ignored detail have yielded local strain measurement, improved angular resolution of lattice orientation, mapping of components of dislocation density, and pseudo-symmetry measurement [1-4].

Recent work is presented which adds to these efforts at detail extraction. Similarly to the previously mentioned advances in high resolution EBSD, the work done by the authors relies primarily on cross-correlation and convolution techniques to measure minute differences within the EBSD patterns. These additional developments include: a software-based approach to pattern center determination, with an order of magnitude improvement over the previous methods; mixed pattern separation in polycrystalline materials for greater effective spatial resolution; and work on grain boundary plane determination from surface scans.

Precise knowledge of pattern center (PC), the geometry of the electron interaction volume at each scan location in a sample in relation to the phosphor detector screen (Fig. 1), is of great importance in high resolution EBSD work, where the new techniques are much more sensitive to uncertainty in the microscope geometry than earlier EBSD methods. Error in pattern center introduces phantom strains and lattice rotations into absolute strain measurements [5, 6]. It can also contribute to error in relative measures (such as deformation gradients) from pattern comparisons when the spatial translation between the compared patterns becomes too great.

A software/image processing methodology for determining accurate pattern center for a given EBSD image was previously reported by the authors, based upon analysis of the geometry of the Kikuchi pattern bands (Fig. 2 and Fig. 3). This presentation discusses the PC determination framework, validation procedures undertaken, and applications.

Furthermore, EBSD image analysis techniques are applied to the recovery of lattice characteristics of mixed grains within the electron interaction volume as a step towards improving spatial resolution. Monte Carlo simulations of electron backscattering interaction volumes are convolved with simulated grain boundaries and the resulting curves (Fig. 4) are compared with the relative fractions of actual mixed pattern EBSD images in line scans [7]. These comparisons are used to extract three-dimensional grain boundary plane information from an EBSD surface scan. Results are compared with actual 3D data obtained using the focused ion beam (FIB) for material removal [8]. 


\section{References}

[1] A.J. Wilkinson, G. Meaden, D.J. Dingley, Ultramicroscopy, 106 (2006) 307-313.

[2] J. Kacher, Cross-Correlation-Based Texture Analysis Using Kinematically Simulated Patterns, in: Mechanical Engineering, Brigham Young University, Provo, 2009, pp. 107.

[3] C.J. Gardner, B.L. Adams, J. Basinger, D.T. Fullwood, International Journal of Plasticity, 26 (2010) 1234-1247.

[4] C.J. Gardner, J. Kacher, J. Basinger, B.L. Adams, M.S. Oztop, J.W. Kysar, Experimental Mechanics, (2010) 1-15.

[5] S. Villert, C. Maurice, C. Wyon, R. Fortunier, J. Microsc., 233 (2009) 290-301.

[6] J. Kacher, C. Landon, B.L. Adams, D. Fullwood, Ultramicroscopy, 109 (2009) 1148-1156.

[7] J. Kacher, B.L. Adams, D. Fullwood, C. Landon, Separating Coincident Electron Backscatter Diffraction Patterns Near interfaces, in: A.D. Rollett (Ed.) Applications of Texture Analysis, John Wiley \& Sons, Hoboken, NJ, 2009, pp. 147-154.

[8] This research was supported by the Army Research Office under David Stepp, contract W911NF0810350.

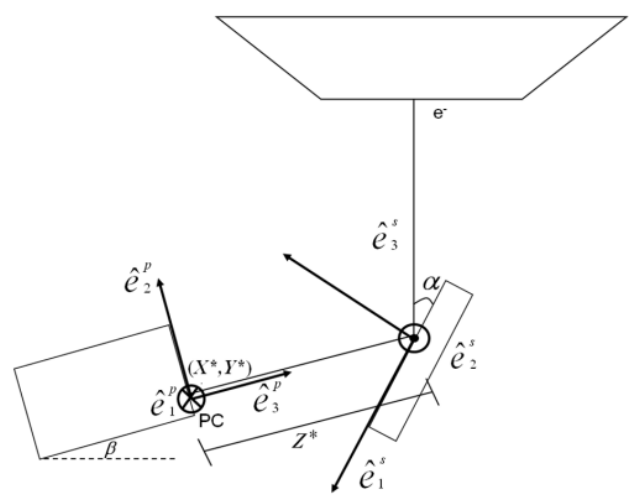

Figure 1: Pattern center geometry. $\mathrm{X}^{*}$ and $\mathrm{Y}^{*}$ are defined relative to the bottom left corner of the phosphor screen. $Z^{*}$ is the magnitude of the normal vector back to the interaction volume on the sample. Units of $P C$ are given as a percent of the screen width.

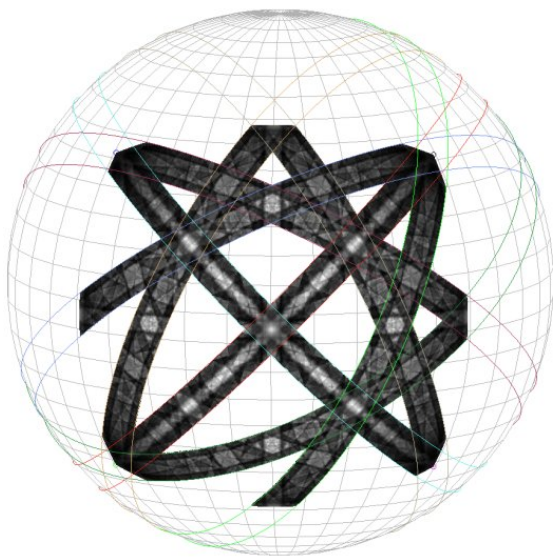

Figure 2: Dynamically simulated iron-alpha EBSD pattern (courtesy of Aimo Winkelmann) shown on a spherical projection about an assumed pattern center. A limited number of Kikuchi bands are examined for band edge parallelism and centering on a great circle.

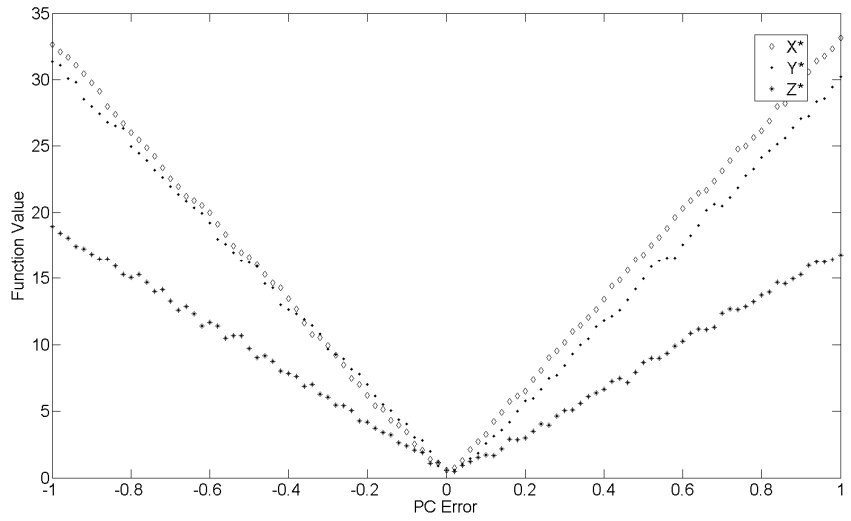

Figure 3: PC search space for a dynamically simulated pattern. In the absence of noise encountered in a real EBSD pattern, the search space is shown to have a global minimum at the correct PC.
Simulated Grain Boundary (60 degree angle) Convolution

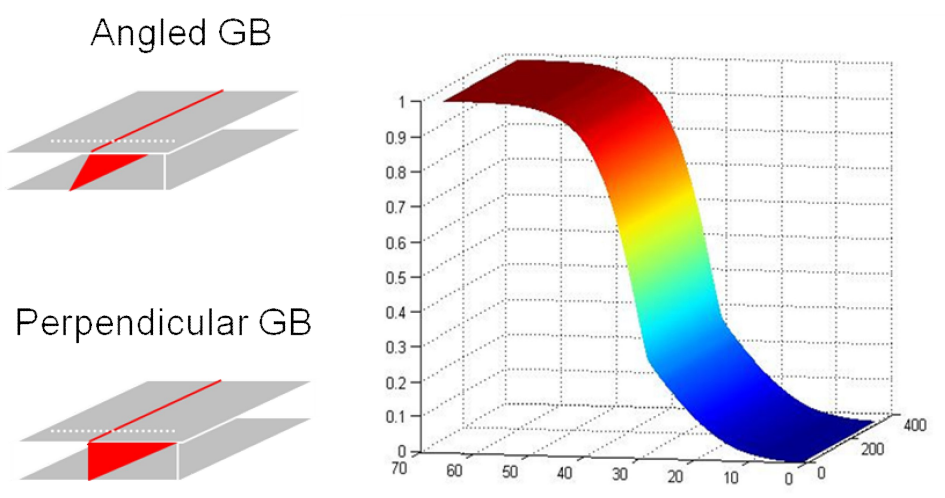

Figure 4: As the electron interaction volume moves across a grain boundary, the relative fraction of one grain's contribution to the mixed pattern can be depicted as a curve, gotten by cross correlation of the mixed pattern with a reference. The curve shown here was generated by the convolution of a Monte Carlo simulated interaction volume with a simulated grain boundary tilted at a 60 degree angle. 